\title{
NIVELES DE INTERVENCION EN EL AULA PARA LA ATENCION DE ESTUDIANTES CON TRASTORNOS DE LA ATENCION
}

\author{
Lic. Carmen Cubero Venegas'
}

\begin{abstract}
RESUMEN: La atención de los estudiantes con trastornos de la atención es un reto para el maestro, ya que este debe responder con estrategias que permitan el desarrollo personal y académico de todos los estudiantes. Planear la manera en que se puede llevar a cabo la tarea docente en una forma que les permita a estos alumnos desenvolverse y aprehender a manejar exitosamente su dificultad y que a la vez, las lecciones fluyan de manera adecuada, es el objetivo principal de este trabajo.
\end{abstract}

La planeación del trabajo académico y el manejo del comportamiento organizado en tres niveles de intervención, permite que el orden y la estructura sean el andamiaje con el cual puedan funcionar estos estudiantes.

Palabras clave: TRASTORNOS DE LA ATENCIÓN/ DÉFICIT ATENCIONAL/NIVELES DE INTERVENCIÓN EN EL AULA/DISCIPLINA/ PLANEAMIENTO DEL TRABAJO DE CLASE/MANEJO DEL COMPORTAMIENTO/

El trabajo docente lleva implícito, entre otros aspectos, las relaciones interpersonales y las acciones de planeación y planificación, por lo que tres de las muchas cualidades que debe desarrollar, revisar e implementar un buen docente, se refieren a:

a) Su capacidad personal de ser un excelente conocedor del desarrollo integral del ser humano y las múltiples formas que existen para su estímulo

b) Sus destrezas para administrar un currículum que sea significativo, retador, innovador, interesante, efectivo y eficiente.

c) Sus habilidades en la interrelación personal; manejo de la comunicación, los límites, las habilidades sociales y la resolución de conflictos propios del aula y de la interacción humana.

\footnotetext{
${ }^{1}$ Licenciada en Psicología de la Universidad de Costa Rica. Profesora de Enseñanza Media de Universidad de Costa Rica. Estudios de Maestría en Administración Pública en el Instituto Centroamericano de Administración Pública. Profesora de la Escuela de Orientación y Educación Especial, Sección de Psicopedagogía de la Universidad de Costa Rica. Subdirectora del IIMEC.ccubero@cariari.ucr.ac.cl
} 
En el caso de las personas con trastornos de la atención, estas cualidades adquieren un significado especial, ya que las características del trastorno demandan al docente un constante manejo de la relación interpersonal, la creación de una estructura curricular que permita la atención de las necesidades educativas especiales y el manejo de la disciplina todo tendiente a proporcionar estrategias de autocontrol para el aula, para el centro educativo y para la vida cotidiana. También, la demanda es mayor en el trabajo y apoyo que requiere la familia del niño y la interconsulta con otros profesionales, entre ellos, médicos, psicólogos y psiquiatras.

En el caso de los estudiantes que presentan trastorno de la atención las habilidades y destrezas señaladas tienen una importancia especial, pues estos estudiantes pueden funcionar muy bien cuando hay una estructura organizativa en el aula y en el centro educativo donde las reglas estén claras, sean consistentes y cuando la organización del trabajo cotidiano tenga un fluir suave, con rutinas establecidas y en donde se preste atención a la forma en que se administran las excepciones propias del trabajo docente.

La asistencia a la escuela de los niños y adolescentes con trastornos de la atención puede ser muy difícil. El éxito escolar significa, entre otras cosas, que el alumno tenga la capacidad de prestar atención, concentrarse y controlar sus conductas de movilidad e impulsividad; justamente las áreas en que estos alumnos tienen mayores dificultades.

Es necesario recordar, los trastornos de la atención o desorden de déficit de atención, como se le conoce, es una condición que hace difícil que la persona pueda estar tranquila, controlar sus impulsos y poner atención. Aunque estas dificultades suelen presentarse antes de los siete años, es en el centro educativo donde la manifestación se hace más notable. La frecuencia de aparición de estos trastornos está indicada alrededor de 5 de cada 100 niños y adolescentes. (Barkley, 1990, citado por García 2001)

Cuando un docente empieza a sospechar que un niño tiene este trastorno, es muy importante que sistematice su observación y preste atención a los momentos y circunstancias en que las conductas se presentan con mayor frecuencia e intensidad. Es necesario, en este caso, que el docente tenga presente que hay tres señales básicas con las cuales puede organizar la información que proviene de su observación, a saber:

A) Cuando los alumnos tienen problemas al poner atención.

B) Cuando los alumnos son muy activos.

C) Cuando los alumnos actúan antes de pensar.

Algunas de las conductas que un docente puede apreciar en sus estudiantes con estos problemas y que podrían ayudar mucho en el diagnóstico posterior del trastorno, 
podrían describirse como se indica líneas abajo. Esta descripción en ningún momento es de carácter diagnóstico, sino ilustrativo, para indicar la forma en que el docente puede organizar la información.

Estudiosos de los trastornos de atención como Barkley (1998), Armstrong (1997 y 2001), García Castaño (2001), Scandar (2000), entre otros, proponen en el caso de los alumnos que tienen dificultades para poner atención, llamados en la terminología diagnóstica como "tipo predominantemente desatento", la persona no puede ni enfocar ni mantener enfocada su atención en una tarea o actividad; a menudo presentan conductas como no prestar atención a los detalles: no mantenerse enfocados en el juego o el trabajo escolar por el tiempo necesario para concluirlo; no seguir las instrucciones aunque sea para una actividad de su interés; con frecuencia no termina el trabajo escolar en el aula o en la casa; manifiesta dificultades para hacer sus tareas solo; el resultado de su trabajo es de baja calidad, pues no son capaces de organizarlo adecuadamente; pueden distraerse fácilmente por detalles o situaciones irrelevantes; pierden cosas tales como sus juguetes, su trabajo escolar o su suéter o zapatos de deportes, o bien, el material con el que tienen que trabajar.

Los alumnos que son muy activos, diagnosticados como personas con trastorno "tipo predominantemente hiperactivo", generalmente son los primeros en ser identificados por el docente como estudiantes que presentan importantes dificultades para cumplir con su trabajo escolar; sus conductas son perturbadoras para el desarrollo adecuado de la actividad académica, permanecen siempre en movimiento; están inquietos y se retuercen en su asiento, se levantan de la silla cuando no deben; corren constantemente; se suben en objetos o sitios inadecuados; presentar dificultades para jugar con tranquilidad; hablan demasiado; hacen ruidos con objetos; tamborilean con el lápiz, guardan y sacan los materiales escolares; esparcen sus objetos personales en el pupitre, en el suelo y hasta en el escritorio del compañero y les cuesta seguir las normas de un juego, entre otras conductas muestran una situación de hiperactividad en un niño.

Los alumnos con conductas de carácter impulsivo casi siempre actúan antes de pensar o reflexionar en o que deben hacer; por ejemplo pueden salir corriendo del aula al oír la campana o cuando el docente dice que es hora del recreo; pueden subirse a un árbol muy alto y no poder bajarse; pueden atravesar una calle sin mirar si lo puede hacer; suelen decir cosas abruptamente sin medir las consecuencias de lo que dijeron; pueden dar una respuesta antes de que se complete la pregunta; tienen dificultad para esperar turno o levantar la mano y acostumbran interrumpir al docente o a los compañeros cuanto estos están hablando. 
También se encuentran los niños que presentan rasgos o conductas de los tipos descritos anteriormente; tienen problemas para poner atención, son hiperactivos y tiene dificultades para controlar sus impulsos, en estos niños estas conductas son la norma y no la excepción, por lo que las dificultades en la casa, en la escuela y con los amigos son parte de su vida cotidiana y la respuesta que reciben del medio ante estas conductas que no puede controlar y que no las presenta intencionalmente, les pueden provocar reacciones de inseguridad, baja autoestima, ansiedad y depresión.

Hay muchas maneras a través de las cuales la escuela y la familia pueden ayudar a estos niños. En este artículo, se proponen niveles de intervención que ayuden al docente a potencializar las cualidades que se privilegiaron en el inicio del presente articulo. Para la atención de las personas con trastornos de atención, se puede trabajar en tres niveles de intervención, inspirado en el modelo propuesto por Charles (1989) para el manejo de clases que disminuyen el mal comportamiento.

\section{Niveles de intervención:}

Como puede apreciarse en la figura 1, los niveles de intervención en el aula son tres: el nivel preventivo, el nivel de apoyo y el nivel correctivo. Estos corresponden a acciones que tienen una interdependencia entre sí. La aplicación de cada uno se da en diferentes momentos de la vida cotidiana. En este documento, se hará énfasis en los beneficios que se pueden obtener a partir de la intervención sistemática en el aula.

a) Nivel preventivo: busca anticipar las acciones para evitar que una conducta se manifieste, o bien, busca las acciones para que la conducta se incorpore o se mantenga. En el caso de los niños con trastornos de la atención (TA) éstos manifiestan conductas que se hace necesario controlar o eliminar, y requieren incorporar otras y mantenerlas, tanto para que tengan éxito en el trabajo académico como en las relaciones interpersonales.

b) Nivel de apoyo: busca que las conductas que se quieren evitar o eliminar sean excluidas mediante acciones de recordatorio o de aplicación directa de consecuencias. Asimismo, las conductas que se desean incorporar o mantener, puedan ser reforzadas oportunamente. Como particularidad, el nivel de apoyo permite que la lección se dé sin interrupción, pues las acciones son recordatorios previamente acordados entre el docente y el estudiante, o entre el grupo, el 
docente y el niño. Aquí se incluye todo refuerzo de carácter afectivo: sonrisas, palmadas suaves en la espalda o sobre la cabeza, caricias, palabras cariñosas, reconocimientos breves, etc.

c) Nivel correctivo: busca enmendar el comportamiento inadecuado y reorientarlo. Nótese que no es solo corregir, pues tanto en el caso de los niños con trastornos en la atención como en el de los que no lo tienen, se corrige la conducta que para el adulto es perturbadora o inadecuada; sin embargo, si no se trabaja la reorientación y/o el modelaje de lo que se requiere, en muchas ocasiones el alumno no sabe qué es lo que se espera de él en ese momento y circunstancia. En caso de que sea una conducta de eliminación o de incorporación que se había prevista en el nivel preventivo y se había apoyado debidamente en el segundo nivel de intervención, entonces lo que cabe en este nivel es la aplicación de la consecuencia establecida, sin dejar por ello de reorientar, informar o recordar cuál es la conducta que se está esperando. En el caso de que sea una conducta inaceptable, no prevista oportunamente, esta debe detenerse de inmediato, aplicar una consecuencia razonable y adecuada a la falta, que deje en claro cuál es la conducta que se espera en otra oportunidad. 
Figura 1

Niveles de intervención en el trabajo cotidiano del aula

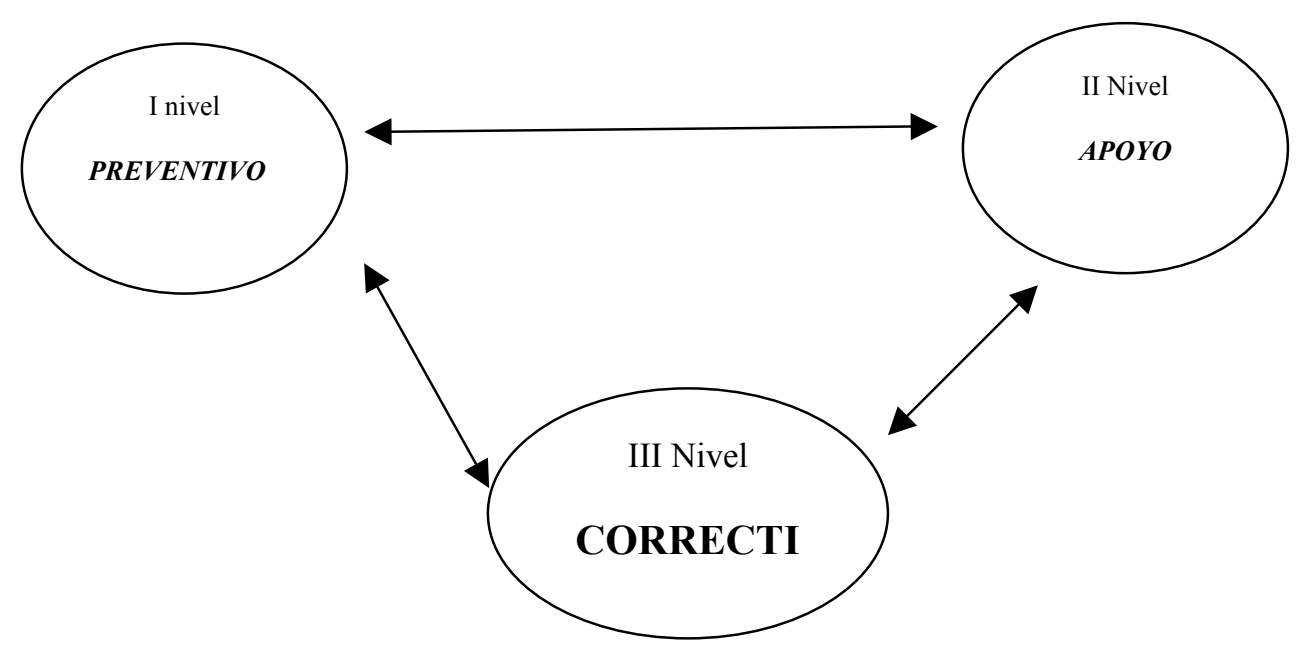

\section{Primer Nivel de Intervención en el aula: Preventivo}

Curwin y Mendler (1983), afirman que en el nivel preventivo, se intenta minimizar o prevenir el surgimiento de problemas en la clase, y organiza seis etapas para la implementación de esta intervención, los cuales para fines de este trabajo, son adaptados por la autora.

1. Aumentar la autoconciencia del docente. Se requiere que el docente se conozca a sí mismo; reconozca sus potencialidades, sus fortalezas, sus necesidades personales, su estilo de enseñar y aprender, tenga una clara concepción de lo que piensa que es su trabajo profesional y su papel en la vida de los alumnos.

2. Aumentar el conocimiento de los estudiantes. El docente necesita conocer quiénes son sus alumnos, por qué y para qué están en el centro educativo. Es importante que reconozca las necesidades de los estudiantes como grupo etario y como individuos. Entre más consciente es el docente de sus alumnos, más eficiente es el trabajo que puede ejecutar y más clara su función de promotor del desarrollo integral.

3. Expresión de los verdaderos sentimientos. Como modelo de comportamiento y de persona y por el innegable papel que cumple el docente en la vida de sus estudiantes, es importante que este clarifique y aprenda a expresar de manera asertiva y sana los sentimientos. Cuando un docente se conoce a sí mismo y saber interpretar lo que está sintiendo, puede comunicarles a los estudiantes sus 
propias necesidades sin entrar en estilos de interacción poco saludables, que generalmente provocan que todos los participantes de la relación salgan lastimados, en especial los niños que tienen una necesidad de atención diferenciada, dadas sus características particulares.

4. Descubrir y reconocer las posibles alternativas o modelos de conducta. El docente que realmente quiere hacer un manejo efectivo de su clase, cuidar su salud mental y favorecer el desarrollo de las potencialidades de los estudiantes, es aquel que piensa y reflexiona sobre cuáles son los comportamientos, las destrezas y habilidades que necesitan sus alumnos para cumplir con las tareas cognoscitivas y poder desenvolverse en un ambiente agradable que permita su expresión personal y la de sus compañeros. Luego de clarificar esto, se detiene a planificar las condiciones personales y ambientales que se requieren para poder llevar a cabo el plan propuesto. Con estas líneas generales, está listo para determinar las acciones que debe implementar en el nivel preventivo, para cumplir con su papel social de ente formador.

5. Establecimiento de contratos sociales. Cualquier acción que se necesite implementar, debe ser previamente planificada para que todos los participantes del acto educativo estén claros de su participación. El compromiso es individual y grupal. Individual porque requiere que el alumno entienda y comprenda la razón de la norma o conducta esperada, y grupal, porque el beneficio es para todos. Cuando se establece un contrato, todas las partes deben estar de acuerdo con las condiciones, y por tanto, todos deben ser partícipes. En el caso que nos ocupa, los contratos sociales son las normas de convivencia cotidiana, y se constituyen en parte del desarrollo de las habilidades sociales que les permiten a los alumnos y al docente cumplir la tarea que vienen a realizar en el Centro Educativo. Por esto, se requiere el compromiso de todos los participantes para poder crear el ambiente de convivencia que favorezca la expresión de la individualidad con el respeto a la colectividad. Las normas que van a regir ese contrato propongo que deben ser, confeccionadas siguiendo el criterio de las tres "c": claras, concretas y concisas, lo que facilitará su comprensión y aplicación. Deben ser expresadas en un lenguaje sencillo y con un vocabulario reconocido por el alumno. En la medida de lo posible, es necesario que sean redactadas en términos positivos que tengan explícitamente determinadas las consecuencias positivas si se cumplen, y las consecuencias negativas si se transgreden. Es importante reiterar que la norma podrá ser 
incorporada siempre y cuando el adulto y el ambiente sean consistentes en su aplicación. Esta es justamente la razón del nivel de intervención preventivo: preparar al maestro para actuar en la forma correcta en caso de que se presente una conducta inadecuada, o bien, si es necesario incorporar una estrategia que permita el desarrollo de las habilidades personales y el derecho de todo el grupo a estar en un ambiente apto para el aprendizaje.

6. Realizar los contratos sociales. Esta etapa es muy importante. La mayoría de las veces, la acción se queda en la etapa anterior y no llega a aplicarse el contrato establecido. La consistencia, como se mencionó líneas arriba, es la que hace la diferencia. El dicho popular "es mejor una onza de prevención que una libra de curación", se aplica en este caso. Cuando se han seguido las etapas anteriores, el docente tiene una serie de recursos que le permiten ser más justo, ecuánime y objetivo en su tarea de corregir y formar seres humanos. Se evita la molesta "reacción" que generalmente responde a criterios de enojo y que dificultan la reflexión positiva ante la situación. La reacción es un proceso mucho más impulsivo que la acción, responde a niveles de defensa y de ataque que son el caldo para enfrascarse en las luchas de poder y la antesala de lastimar la autoestima de las personas. Cualquier adulto que va a estar en constante relación con niños y adolescentes, requiere el desarrollo de destrezas que le permitan actuar y no reaccionar. La ejecución de los contratos va en esta línea; permite prever y actuar con mayor tranquilidad y por tanto, las acciones estarán orientadas al desarrollo de un locus de control interno y no externo, que es el que propicia la reacción.

En el nivel preventivo, el objetivo es siempre prevenir los comportamientos antes que lidiar con ellos, por lo que el ambiente debe organizarse, así como el proceso educativo para facilitar el éxito de los participantes en él.

Yelon y Weinstein (1988, p: 397-398) ofrecen una serie de sugerencias para que al docente se le facilite la ejecución del nivel de intervención preventivo, y que en el caso de los niños con trastornos de atención, cobran una mayor relevancia, por cuanto estos niños necesitan de un ambiente estructurado y de una persona consistente, que de apoyo, seguridad y confianza; la autora ha realizado una adaptación de las mismas para el caso que ocupa este artículo, entre las sugerencias señaladas están: 
- Comportarse con firmeza y seriedad. Los niños con conductas provocadas por el trastorno de atención, sea este con hiperactividad o sin ella, requieren de adultos que sean firmes en sus actos, serios en su planteamiento y con una gran dosis de paciencia y cariño. La estabilidad en la relación que provoca esta conducta favorece que el niño discrimine que el docente es su aliado y no su enemigo, que el ambiente en que está es seguro y que es comprendido y aceptado.

- Estar preparado para todo. Se ha dicho que lo más constante en los niños con trastornos de atención es el cambio. Esta paradoja es cierta; no es posible predecir cual va a ser la reacción o la conducta que puede presentar el niño, ni tampoco se sabe si la va a incorporar a su estilo de comportamiento cotidiano, es por esta razón que el docente debe saber que el niño, debido a las características de su trastorno, puede persistir en una conducta por algún tiempo, dejarla y luego retomarla, o salir con una respuesta inadecuada o diferente a la esperada por el docente. Estar preparado para estas situaciones permite la acción y no la reacción del docente. Es necesario tener presente que ninguna conducta inadecuada se inicia a partir de las malas intenciones de las personas; es generalmente la dinámica que se dé la que marca la diferencia.

- Hacer siempre interesante la lección. Dar sorpresas o utilizar algún elemento de innovación, particularmente al introducir el tema. Aprender a distinguir el clima de la clase conforme avanza la explicación del tema para presentar algún elemento que permita volver a capturar la atención, o bien, reconocer que la materia es complicada, abstracta para el nivel de desarrollo cognitivo de los estudiantes o árida, favorece que se tomen las acciones preventivas y que se esté listo para actuar en el momento que se note descontento o desconcierto por parte de los estudiantes.

- Tener muy claro cuándo debe prevalecer el criterio del docente. En este sentido es importante que el docente sepa distinguir entre la intencionalidad, la argumentación y la insolencia. En algunas ocasiones, la intencionalidad con que un alumno presenta una conducta marca la diferencia en la aplicación de las consecuencias. La intencionalidad debe ser intuida por el docente; este debe conocer a los estudiantes y discriminar las intenciones que tuvo para actuar de una manera particular. En el caso de los niños con trastornos de la atención, se encuentran una serie de conductas que a simple vista parecieran ser realizadas con mala intención, ejemplo de ellas, no seguir las instrucciones cuando se 
acaban de dar o repetir, interrumpir a otros, decir lo que piensan sin usar ningún filtro social, etc.; en el caso de un cuadro dominado por la impulsividad, o bien pararse del asiento en el momento más inoportuno, jugar con cualquier objeto, hacer ruidos, etc.; en el caso de un cuadro dominado por la hiperactividad o dejar algunas preguntas de un examen sin contestar o dar una respuesta pobre o simple en el caso de un dominio de la inatención. Si se conocen las características del niño y las del trastorno que presenta, entonces se puede discriminar que no hay mala intención sino una falta de autocontrol, que es, en última instancia, lo que requiere que se trabaje con el niño.

La argumentación es otro aspecto importante; a él se refiere cuando hay que discriminar entre un acto matizado por la discusión propia de una lucha de poder entre el docente y el alumno, o entre un alumno y otro, y cuando lo que el niño está tratando de hacer es buscar una argumentación que explique su conducta. En este caso, es necesario darle al niño la oportunidad de que "arme" esa argumentación y la pueda comunicar adecuadamente, aunque después se puede estar de acuerdo con ella o no. Por último, la insolencia es un asunto que no se debe permitir, y que en muchas ocasiones se suele confundir con el humor; sin embargo, debe tenerse presente que una cosa es una ocurrencia graciosa, y otra, un acto intencional que busca la desautorización del docente y su trabajo profesional. El humor es un factor protector que permite un momento de camaradería y relajación en el aula y que va a fortalecer la resiliencia; la insolencia, en cambio, es un acto que no debe permitírsele a ninguna persona.

En el caso de los niños con déficit de atención, se hace necesario el trabajo en las habilidades sociales que les permitan incorporar destrezas que mejoren su convivencia, especialmente, en aquellos que presentan conductas dominadas por la impulsividad y por la hiperactividad, que favorecen el rechazo de los otros niños que sienten sus derechos pisoteados. Es necesario tener presente que estos niños no tienen mala intención, solamente que se les dificulta usar las normas de convivencia que suelen acatar sus iguales en forma natural. Estos niños deben desarrollar esas normas mediante un proceso de enseñanza - aprendizaje que requiere de paciencia, esfuerzo y sobretodo, consistencia.

- Ser congruente. La congruencia es una de las cualidades más codiciables en un adulto que tenga que participar en la crianza de los niños, y adquiere particular utilidad cuando se trabaja con niños con trastornos de la atención. En el nivel de 
intervención preventiva y, como se mencionó anteriormente, la congruencia permite la incorporación de las normas y las rutinas que tanto favorecen el trabajo en el aula y que son uno de los puntos más vulnerables en los niños con estos trastornos.

- Ser justo. La justicia es un valor que reclaman los niños y todas las personas, pero particularmente aquellos que requieren la atención de una necesidad educativa especial como es el caso de los niños que presentan trastornos de la atención. En la lectura del libro "Hiperactivo, impulsivo, distraído ¿Me conoces?", el Dr. José Bauermaister (2000, p:231), se refiere a la justicia en términos educativos:

" Quizá el educador que está leyendo estas líneas pensará, si no lo ha hecho anteriormente que la práctica de modificar los requerimientos es injusta, ya que no se les está exigiendo a todos los estudiantes por igual. El diccionario de la lengua española define justicia como 'una de las cuatro virtudes cardinales, que inclina a dar a cada uno lo que le corresponde o pertenece'. ¿No cree usted que la vulnerabilidad biopsicosocial que predispone a un niño a tener dificultades para sostener la atención, autocontrolarse, dominar la lectura o escribir con rapidez o eficiencia deber ser razón para 'inclinarse a dar a cada uno lo que le corresponde'? ¿Qué práctica seguimos cuando tenemos un estudiante con limitaciones visuales o impedimento físico? ¿Acaso no hacemos justicia con ellos debido a su condición? ... sabemos que cada estudiante es diferente. Por consiguiente, lo que es posiblemente injusto es tratarlos como si fueran iguales. "

Es necesario desde la perspectiva preventiva, prestar atención, tal y como lo plantea Kounin (citado por Charles, 1989) a la importancia de cuidar el movimiento de la lección, el ritmo, la velocidad y las transiciones, para así, mantener a los alumnos atentos. Aquellos docentes que pueden cambiar con suavidad de una actividad a otra, que pueden mantener un ritmo adecuado a dichas actividades, y una velocidad sostenida, pueden captar mejor la atención de los alumnos. Especial mención merecen para este autor, las transiciones, por cuanto un docente que tenga dificultad en el manejo de la transición, provoca en el alumno confusión, actividad innecesaria, ruidos, atrasos y por supuesto, mala conducta; en el caso de los niños con trastornos de la atención, provoca aún más dificultad, porque la facilidad 
que tienen para distraerse aumenta el riesgo de que no puedan autocontrolar su conducta cuando el docente requiere de su participación en la nueva actividad.

En este nivel de intervención preventivo la comunicación directamente dirigida a la situación y no al alumno, requiere un señalamiento especial. La comunicación congruente, tal y como la señala Ginott (citado por Charles, 1989), es el factor crucial en el ambiente de clase y en la relación interpersonal. Es una manera armoniosa y auténtica de hablar en la que los mensajes de los docentes se ajustan a los sentimientos de los alumnos, lo que para los niños con trastornos de la atención es fundamental. En la comunicación congruente, el docente sabe que no debe negar sus propios sentimientos ni los de los alumnos, pero sabe bien cómo manejar y expresar su enojo en forma razonable, y así, no lastima la autoestima de nadie. Cuando el docente se siente molesto puede expresar su enojo, según lo citan Cubero, Abarca y Nieto (1996, p: 22) " en forma breve y firmemente, en primera persona: 'Estoy enojado" y no en tercera persona: 'No se ha portado bien', porque de esta manera estaría atacando al estudiante y no la situación".

\section{Segundo Nivel de Intervención en el aula: Apoyo}

En el nivel de intervención de apoyo se busca que el alumno se comporte como se necesita en ese momento. Se basa sobre todo en el lenguaje corporal del docente y en una estrategia de comunicación asertiva muy breve, cuando la situación lo requiere.

El lenguaje corporal involucra contacto visual, proximidad física, desplazamiento en el aula, expresión facial, gestos, señales como ponerse el dedo en los labios o mover la cabeza en señal de desaprobación; implica también sonrisas y expresiones que indican aprobación, como una forma de reforzar el comportamiento adecuado cuando el alumno está haciendo lo que se espera que haga. El lenguaje no verbal expresa al alumno los sentimientos e intenciones del docente; pueden comunicar alegría, enojo, seriedad, entusiasmo, desorden, o apatía, y por esto, deben ser dominadas por el docente mediante técnicas de autoconocimiento.

Jones (citado por Charles, 1989) recomienda usar el lenguaje no verbal, especialmente las expresiones faciales, ya que este le permite recordar al alumno que se espera de él una conducta diferente sin tener que interrumpir la lección.

Las señales que se usen para hacer estos recordatorios deben ser de mutuo conocimiento por parte del alumno y del maestro, o mejor, podrían formar parte del contrato de comportamiento que se puede hacer con los estudiantes en la intervención preventiva. Es importante recordar que en el caso de los estudiantes con trastornos de la atención, el 
recordatorio de que su conducta se está desviando de lo esperado, debe ser practicado para que lo pueda incorporar como parte de su trabajo en el aula.

Siempre en el nivel de intervención de apoyo, se pretende ayudar al estudiante a mantener su autocontrol, por lo que deben ser aplicadas en el momento en que la conducta inadecuada se empieza a presentar.

En el caso de los niños con trastornos de atención, se puede requerir que el docente reestructure el trabajo para que se adapte a las necesidades de atención, concentración y tiempo. Es conveniente que el docente esté pendiente del rendimiento y ritmo de trabajo que tiene el estudiante ante determinada tarea y pueda darle el apoyo necesario para que lo cumpla con éxito; el uso de claves o sugerencias, tanto en el trabajo cotidiano como en las pruebas escritas, puede ser muy útil para mantener enfocado al estudiante en el contenido, actividad o material con el que se está trabajando. Es importante tomar conciencia que estos estudiantes, al recibir el apoyo del docente, se sienten estimulados, respetados y por tanto, tienden a tener un mejor rendimiento en su trabajo; asimismo, por la dificultad que tienen para permanecer mucho tiempo en la misma tarea, el reenfocarlos con alguna pista o con una reestructuración no significativa de la misma, les permite entusiasmarse por cumplir lo asignado. Por otro lado, el estar pendiente y darle claves, no es sinónimo de "soplarle" el resultado, es proporcionarle ayuda para que pueda continuar. Es necesario tener presente que, al igual que otros niños, enfrentar una pregunta o una asignación requiere una interpretación de las instrucciones, sean escritas o verbales, y al mediar esta interpretación, se entra en un plano subjetivo entre el emisor del mensaje, el mensaje mismo y el receptor. En cualquiera de estos puntos, se puede presentar una interpretación inadecuada que en muchas ocasiones no permite que el estudiante demuestre todo el conocimiento que tiene sobre el asunto. Observar su conducta durante el trabajo o el examen, preguntarle lo que entendió de la instrucción, recordarle algún dato o circunstancia especial que sucedió cuando se estudió el tema o lo que había en el texto o en la actividad innovadora, puede ser suficiente para que el estudiante encuentre la respuesta, que en la mayoría de los casos, ya conocía.

Se sigue pensando que incorporar objetos diversos en el aula no es lo más conveniente para los niños que tienen dificultades para enfocar su atención y para comprender las instrucciones o explicaciones que se les está dando. No obstante, se ha visto a niños con trastornos de atención funcionar muy bien en ambientes educativos ricos en estímulos visuales, auditivos y táctiles, que les ofrecen la oportunidad de apreciar los contenidos desde una perspectiva multisensorial. 
Un recurso valioso para reenfocar la atención, es desplazarse hacia el lugar que ocupa el estudiante y mirarlo a los ojos, darle la explicación o hablarle dirigiéndose a él, tocarlo suavemente en el hombro o tocar sus materiales y recordar en forma general lo que se espera que hagan los niños en ese momento.

En el segundo nivel de intervención en el aula, el refuerzo positivo a las conductas esperadas que contrarresten las propias de la inatención, la hiperactividad y la impulsividad, son necesarias. Estos refuerzos que se han clarificado en el nivel de intervención preventiva, pueden ser aplicados en el momento en que se manifieste la conducta esperada, o bien, cuando no se está presentando la conducta inadecuada.

Este nivel de intervención requiere la exigencia de un buen comportamiento; el estudiante debe saber que es lo que se espera de él y no es el momento de "negociar" otros comportamientos. Posteriormente, se pueden revisar las estrategias de apoyo que se han diseñado, si estas no han dado resultado, en este momento lo que procede es aplicar el apoyo, y si no se tiene éxito en el recordatorio de la conducta esperada, se debe pasar al tercer nivel de intervención, que es el correctivo.

Aceptar que los estudiantes están cansados, aburridos, que el contenido presenta dificultades, etc., es apropiado; no obstante, esto no significa que se debe dejar de realizar, al contrario, este reconocimiento de los sentimientos humaniza el aula, porque permite el trabajo práctico de valores como la responsabilidad (al tener que terminar el trabajo), el respeto (al trabajo), y la tolerancia (hacia los otros y hacia él mismo), etc.

\section{Tercer Nivel de Intervención en el Aula: Correctivo.}

Es bien sabido que por más que se trabajen los dos niveles anteriores, siempre existirán conflictos en el aula debido a que en ella interactúan múltiples personalidades que tienen intereses, patrones de crianza, expectativas, deseos y necesidades diferentes. Esta ha sido la razón por la cual se ha aceptado que se debe trabajar de tal manera que el control del aula y de lo que en ella sucede permita poco espacio a la improvisación, sobretodo cuando se habla de eliminar, incorporar o mantener conductas.

La idea es que la dinámica del aula lleve a la situación de "ganar - ganar", por lo que se pretende que todos tengan satisfechas la mayor cantidad de necesidades posibles, sin detrimento de las necesidades de los otros, particularmente cuando se está pensando en los niños con trastornos de atención y otras necesidades educativas especiales, y cuando se piensa en el docente como persona. 
La idea de ganar - ganar, lleva implícito un buen manejo de los dos niveles anteriores; sin embargo, aún en este tercer nivel no se debe dejar de lado. La estrategia correctiva busca eliminar las conductas que no son adecuadas y reorientarlas, acción que les corresponde a los docentes, o bien a los padres de familia y otros adultos que tienen relación con los niños.

Se debe partir de la idea de que el alumno asume las consecuencias de las conductas inadecuadas que se habían definido, clarificado y aceptado con anterioridad. Las consecuencias deben ser establecidas en el primer nivel de intervención; no deben lastimar la integridad física y psicológica del estudiante, antes bien, deben preservar el cuido de la autoestima, la calidad de vida y el respeto a los derechos humanos. Las consecuencias, por tanto, deben ser escogidas teniendo en consideración la edad del niño, el tipo de conducta inadecuada, la equidad entre la falta y la consecuencia y el momento oportuno para su aplicación. Es necesario estar siempre seguro de que se va a poder aplicar sin importar el momento histórico que se está viviendo, por ejemplo, no se puede poner como consecuencia, que se prive al niño de una excursión escolar, a la que toda la escuela va a asistir, porque ¿quién se va a hacer cargo del niño? Y no se le puede decir simplemente que no venga a la escuela.

La reorientación de la conducta merece una llamada de atención a los adultos que son responsables de la atención de los niños; esto implica que el alumno pueda entender qué es lo que se espera de él y qué puede hacer para conseguirlo; incluso a veces es conveniente modelar la conducta. En el caso de los niños con trastornos de la atención, esta parte de la corrección es fundamental debido a la necesidad que tienen de incorporar, mediante actos repetitivos, algunas conductas, particularmente las de convivencia social. Hay que recordar que estos alumnos tienen dificultades importantes en aspectos relacionados con el autocontrol; son impulsivos en sus acciones, palabras y movimientos, lo que provoca que sean muy vulnerables a romper las reglas establecidas o a presentar conductas que pueden dañarlos a ellos mismos o a otros; por esta razón, es necesario no olvidar esta parte.

\section{Ejemplo práctico de los niveles de intervención en el aula}

En el cuadro 1 se establece una demostración de los niveles de intervención aplicados a la conducta de esperar turno para participar en el aula; conducta que dicho sea de paso, debido a su impulsividad y a las manifestaciones de la hiperactividad, es muy común en los niños que presentan trastornos de la atención. A continuación, se ofrecen 
estrategias que pueden ser aplicadas a la incorporación y mantenimiento de la conducta de esperar turno y estrategias que no necesariamente llevan a la eliminación de la conducta de no seguir las instrucciones para participar en el aula. Nótese que las estrategias se aplican en forma positiva.

\section{A manera de conclusión}

Es innegable la importancia de que el docente aprenda más acerca de los trastornos de la atención de los alumnos para que les pueda dar una respuesta acertada a sus necesidades. La comprensión del hecho de que las conductas que presentan estos niños no obedecen a su intencionalidad o a lo que hagan o no hagan los padres o los mismos docentes, es indispensable para que esa respuesta esté centrada en el marco adecuado. La atención multimodal, con la participación de profesionales, entre ellos el docente, jugando un papel preponderante en la atención y búsqueda de estrategias que faciliten los procesos de enseñanza, de aprendizaje y de autocontrol de las conductas, es básica si se quiere ofrecer a estos niños una mejor calidad de vida en el presente $y$, desarrollar en ellos factores protectores que fortalezcan su resiliencia para enfrentar la adversidad que siempre va a rondar sus vidas, dado que estos trastornos no se curan, forman parte de la estructura cognitiva y de comportamiento de estas personas y por tanto, lo que se puede hacer es darles las mejores herramientas para que fortalezcan sus áreas fuertes y puedan compensar adecuadamente sus debilidades. 
Cuadro 1

Ejemplo de la aplicación de los niveles de intervención en el aula

Conducta: Esperar turno para participar en una discusión en el aula

\begin{tabular}{|c|c|c|c|}
\hline Nivel & $\begin{array}{l}\text { Requerimiento } \\
\text { para el niño }\end{array}$ & Requerimiento para el maestro & Estrategia para incorporar la conducta \\
\hline $\begin{array}{lrr}\text { Preventivo: } & \text { Busca } \\
\text { anticipar las } & \text { acciones } \\
\text { para evitar } & \text { que } & \text { una } \\
\text { conducta } & \text { no } & \text { se } \\
\text { manifieste, o bien, } & \text { o } \\
\text { que se incorpore en el } \\
\text { autocontrol de } & \text { los } \\
\text { alumnos. }\end{array}$ & $\begin{array}{l}\text { Levantar la mano para indicar que } \\
\text { se quiere participar y esperar que } \\
\text { se le ofrezca la palabra para } \\
\text { participar. }\end{array}$ & $\begin{array}{l}\text { Planear cómo debe dar las instrucciones y } \\
\text { cómo debe comunicarlas para que el } \\
\text { alumno pueda esperar turno y planificar la } \\
\text { forma en que puede aplicar las } \\
\text { consecuencias positivas o las negativas en } \\
\text { caso necesario. El docente recuerda que } \\
\text { en el caso de los alumnos con déficit de } \\
\text { atención, no pueden esperar mucho } \\
\text { tiempo, pues tienden a distraerse u olvidar } \\
\text { lo que iban a decir, o bien, la impulsividad } \\
\text { gana la partida, y aún con la mano } \\
\text { levantada, puede interrumpir al otro. }\end{array}$ & $\begin{array}{l}\text { La maestra dice: "Voy a hacer una pregunta y } \\
\text { espero que levanten la mano y esperen turno } \\
\text { para contestar cuando yo digo su nombre" A } \\
\text { continuación hace la pregunta, espera que los } \\
\text { alumnos levanten la mano y empieza a } \\
\text { permitir la participación llamándolos por su } \\
\text { nombre. }\end{array}$ \\
\hline $\begin{array}{l}\text { Apoyo: Busca facilitar } \\
\text { que el alumno se } \\
\text { autocontrole } \\
\text { presente la conducta } \\
\text { esperada }\end{array}$ & $\begin{array}{l}\text { Mantener la mano levantada y } \\
\text { esperar que la maestra diga su } \\
\text { nombre, }\end{array}$ & $\begin{array}{l}\text { Esperar un tiempo prudencial para darle la } \\
\text { palabra, utilizar gestos como una sonrisa, } \\
\text { movimiento de la cabeza para indicar } \\
\text { aprobación, acercamiento físico, } \\
\text { reconocerle brevemente la emisión de la } \\
\text { conducta adecuada. Recordar que no } \\
\text { puede dejarlo mucho rato con la mano } \\
\text { levantada. }\end{array}$ & $\begin{array}{l}\text { Con una sonrisa sincera decirle: "Muy bien, } \\
\text { esperaste bastante tiempo y ahora podes dar } \\
\text { la respuesta", seguir afirmando con la cabeza } \\
\text { la respuesta correcta, corregirla respuesta en } \\
\text { caso de que sea necesario. }\end{array}$ \\
\hline $\begin{array}{lr}\text { Correctivo: } & \text { Busca } \\
\text { eliminar la } & \text { conducta } \\
\text { inadecuada } & \text { y } \\
\text { reorientar al alumno } \\
\text { ofreciéndole } \\
\text { oportunidad de buscar } \\
\text { otras alternativas para } \\
\text { manifestarse. }\end{array}$ & $\begin{array}{l}\text { Eliminar la conducta de hablar sin } \\
\text { esperar turno y apreciar con la } \\
\text { guía del docente, el modelo de } \\
\text { cómo puede hacerlo. }\end{array}$ & $\begin{array}{l}\text { Aplicar la consecuencia negativa. Estar } \\
\text { claro de la forma en que se puede modelar } \\
\text { la conducta esperada y ayudar a que } \\
\text { comprenda los pasos que se requieren } \\
\text { para incorporarla en su repertorio. }\end{array}$ & $\begin{array}{l}\text { La consecuencia negativa puede ser decirle } \\
\text { con firmeza y afecto "No te voy a permitir } \\
\text { hablar, hasta tanto no tengas la mano } \\
\text { levantada y esperes que te dé la palabra". } \\
\text { Posteriormente, puede llamarlo aparte y } \\
\text { decirle: "Es importante para mí que participes } \\
\text { en la clase, pero debes levantar la mano y } \\
\text { mantenerla levantada hasta que te dé la } \\
\text { palabra. Voy a procurar atender tu petición lo } \\
\text { antes posible para que podas participar igual } \\
\text { que tus compañeros". }\end{array}$ \\
\hline
\end{tabular}




\section{Referencias}

American Psychiatric Association. (1995). DSM-IV: Manual de Diagnóstico y Estadística de los Trastornos Mentales. Versión en español Barcelona: Masson

Armostrong, Thomas. (1997). The Myth of the A:D:D: Child: 50 ways to improve your child's behavior and attention span without drugs, labels, or coercion. USA: A Plume Book.

Armstrong, Thomas. (2001). Síndrome de Déficit de Atención con o sin Hiperactividad $A D D / A D H D$. Estrategias en el aula. Argentina: Paidos.

Barkley, Russel. (1998). Attention Deficit Hyperactivity Disorder: A Hnadbook for Diagnosis and Treatment. New York: Guilford.

(1997). ADHD and the nature of self - control. USA: Guilford Press.

Bauermeister, José. (2000). Hiperactivo, impulsivo, distraído ¿Me conoces?. Guía acerca del déficit de atención para padres, maestros y profesionales. Puerto Rico: Atención Inc.

Charles C.M. (1989). Building classroom discipline. (3rd. ed) New York: Longman Inc.

Cubero C. y Nieto M. (2001). Niveles de disciplina. Curso Manejo de la Disciplina en el Aula y en el centro educativo. Costa Rica: Universidad de Costa Rica.

Cubero C, Abarca A y Nieto M. (1996). Percepción y Manejo de la Disciplina en el Aula. Informe de Investigación. Costa Rica: Instituto para el Mejoramiento de la Educación Costarricense. Universidad de Costa Rica.

Curwin R. y Mendler A. (1983). La disciplina en clase. Guía para la organización de la escuela y el aula. Madrid: Narcea S.A. de Ediciones.

García Castaño, Fernando. (2001). Es TDAH y ahora ¿qué?. Trastorno de Déficit de atención/hiperactividad. Una guía básica. San Juan, Puerto Rico: Ediciones Hispalis.

Luna Elizarrarás M.E. (1994). Los alumnos y la normatividad en el salón de clase: una construcción colectiva. Ponencia presentada en el Congreso de Investigación Etnográfica. Costa Rica: Universidad de Costa Rica.

Montcusí S. (s.f.). La vida cotidiana en el aula. Cuadernos de Pedagogía. 183, 43-44

National Institute of Mental Health. (2001). Trastorno hiperactivo de déficit de atención. http://www.nimh.nih.gob/publicat/spadhd.htm.

Rief, Sandra. (1998). ADD/ADHD Checklist. An easy reference for parents \& Teachers. USA: Prentice Hall.

Scandar, Rubén. (2000). El niño que no podía dejar de portarse mal. TDAH: su comprensión y tratamiento. Argentina: Distal.

Wielkiewiez R. M. (1992). Manejo conductual en las escuelas. Principios y métodos. México: Limusa.

Yelon, S. y Weinstein, G. (1988). La psicología en el aula. México: Trillas. 